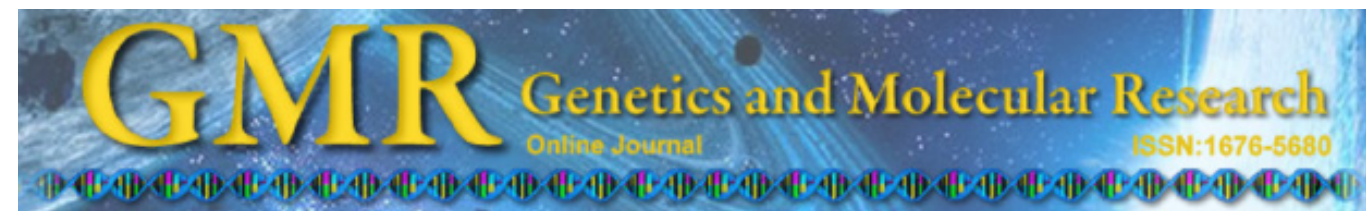

\title{
Molecular diversity analysis of eggplant (Solanum melongena) genetic resources
}

\author{
Z. Ali ${ }^{1,2 *}$, Z.L. Xu ${ }^{1 *}$, D.Y. Zhang ${ }^{1}$, X.L. He ${ }^{1}$, S. Bahadur ${ }^{3}$ and J.X. Yi ${ }^{1}$ \\ ${ }^{1}$ Institute of Agro-Biotechnology, Jiangsu Academy of Agricultural Sciences, \\ Nanjing, China \\ ${ }^{2}$ Department of Plant Breeding and Genetics, University of Agriculture, \\ Faisalabad, Pakistan \\ ${ }^{3}$ Nursery Division, Desert Group, Dubai, United Arab Emirates \\ *These authors contributed equally to this study. \\ Corresponding author: J.X. Yi \\ E-mail: yij@jaas.ac.cn
}

Genet. Mol. Res. 10 (2): 1141-1155 (2011)

Received January 19, 2011

Accepted February 14, 2011

Published June 14, 2011

DOI 10.4238/vol10-2gmr1279

This study was sponsored by the National Science Foundation of China (\#30971798) and the Jiangsu Natural Science Foundation, China (\#BK2010474).

\begin{abstract}
Eggplant (Solanum melongena), a vegetable that is cultivated worldwide, is of considerable importance to agriculture in China. We analyzed the diversity of this plant using inter-simple sequence repeat (ISSR) and RAPD procedures to subdivide 143 Chinese-cultivated eggplants based on coefficient of parentage, genetic diversity index (GDI) and canonical discriminant analysis. ISSR markers were more effective than RAPD markers for detecting genetic diversity, which ranged from $0.10-0.51$, slightly lower than what is known from other crops. Our ISSR/ RAPD data provide molecular evidence that coincides with morphological-based classification into three varieties and further subdivision into eight groups, except for two groups. Intensive use of elite parents and extensive crossing within groups have resulted in increased coefficient of parentage and proportional contribution but decreased GDI during the past decades. The mean coefficient of parentage and proportional con-
\end{abstract}


tribution increased from 0.05 to $0.10 \%$ and from 3.22 to $6.46 \%$ during 1980-1991 and 1992-2003, respectively. The GDI of landraces was 0.21, higher than the 0.09 and 0.08 calculated for the hybrid cultivars released during the two periods. The recent introduction of alien genotypes into eggplant breeding programs may broaden the genetic base.

Key words: Inter-simple squence repeat (ISSR); RAPD; Coefficient of parentage; Genetic diversity index (GDI); Morphometric analysis; Canonical discriminant analysis

\section{INTRODUCTION}

Eggplant (Solanum melongena L.), also known as brinjal or aubergine, is an important Solanaceous vegetable widely consumed in Asia, Europe, Africa, and America. However, the identity, taxonomic status and relationships between varieties involved in cultivated eggplant and its morphologically similar wild/weedy taxa found in India have remained highly confused. Breeders have continually retained interest in the grouping of the germplasm and the pedigree of hybrid cultivars since this information might be particularly helpful determining the most effective breeding strategies.

Eggplant hybrid cultivars and wild and weedy forms are found in India and Indochina as "groups" in the S. melongena complex, in which S. insanum has been regarded as the progenitor of cultivated eggplant (Lester and Hasan, 1991). The cultivated eggplant gerplasm, including a wide range of landraces and hybrid cultivars, were divided into three varieties based on fruit shape: var. depressum, dwarf, early types; var. esculenta, round or egg-shaped fruits, and var. serpentinum, long slender forms (Choudhury, 1976). The species is widely distributed in Asia, Europe, North America, and Africa. To date, a large number of cultivated eggplant germplasm, including all three varieties, have been collected in some countries, e.g., in China (Yi, 2000), Europe and the United States Department of Agriculture (USDA) (Martin and Rhodes, 1979), the EGGNET project at the European level (Polignano et al., 2010), etc. The morphological variations were characterized in the USDA eggplant germplasm collection, and the germplasm was classified into 11 groups (Martin and Rhodes, 1979). In the China collection, a further subdivision of var. esculenta into 3 groups and var. serpentinum into 4 groups were suggested on the basis of 22 morphological traits in particular leaf and fruit characteristics (Yi and Yang, 1997).

In recent years, despite distinct morphological diversity, a close relationship was observed between cultivated $S$. melongena and weedy ( $S$. insanum) and wild (S. incanum) forms in South Asia. A close phylogenetic relationship was suggested among $29 \mathrm{~S}$. melongena, 33 weedy $S$. insanum and 2 wild $S$. incanum accessions based on the analysis of 29 isozyme loci (Karihaloo and Gottlieb, 1995). The conspecific relationship was further confirmed by random amplified polymorphic DNA (RAPD) marker data over 52 accessions (Karihaloo et al., 1995). This may raise breeder's concern, since genetic diversity provides the genetic base for crop enhancement of environmental adaptation, yield or disease resistance. The presence of adequate diverse genetic origin between parents of an $\mathrm{F}_{1}$ hybrid cultivar is critically important (Fasoula and Fasoula, 2002). Moreover, the genetic progress in a breeding program is actually dependent on the variation in the gene pool (Dreisigacker et al., 2004). However, the released Chinese eggplant hybrid cultivars in past decades mainly 
concentrated on less than 15 elite landraces, resulting in a narrow genetic diversity among released hybrid cultivars, similar to decrement of genetic diversity in some other crops, for instance, soybean hybrid cultivars in the USA and Japan caused by intensive breeding programs (Sneller, 1994; Zhou et al., 2000).

The coefficient of parentage (COP) and genetic diversity index (GDI) have usually been employed as morphological-based and indirect measures to estimate the genetic diversity among genotypes (Cowen, 1985; Cox and Snell, 1989). Nevertheless, molecular markers have been widely used in recent years for demonstrating genetic diversity over germplasm collections or breeding populations, providing more direct and powerful solutions at the DNA level. Frequently used molecular markers in genetic diversity estimation include the AFLPs, RFLPs, SSRs, RAPDs, and allozymes in various Solanum species (Karihaloo and Gottlieb, 1995; Karihaloo et al., 1995; Sakata and Lester, 1997; Mace et al., 1999). However, polymerase chain reaction (PCR)-based inter-simple sequence repeat (ISSR) and RAPD markers are particularly useful since automation for high-throughput genotyping is available and no sequence information is required. Most recent use of molecular markers like RAPD and ISSR was reported in broccoli (Lu et al., 2009), ISSR in Indian or sacred lotus, Nelumbo nucifera, (Han et al., 2009), in Primula apennina (Crema et al., 2009), in Cruciferae (Zhang and Dai, 2009), and in Pteroceltis tatarinowii (Chai et al., 2010) to establish genetic diversity and genetic structure of the species. All aforementioned reports on Solanum species mainly focused on revealing the genetic diversity and relationship among the species; however, the molecular marker-based genetic variation among germplasm accessions and released hybrid cultivars and the molecular evidence for subdivision within cultivated eggplant still remain unclear.

The objectives of this study were: 1) to subdivide and trace the pedigree diversity within and among varieties/groups in Chinese-cultivated eggplant collection using ISSR/ RAPD data; 2) to explore the impacts of breeding on divergence of the Chinese-cultivated eggplant.

\section{MATERIAL AND METHODS}

\section{Plant materials}

A total of 143 cultivated eggplant accessions, including 106 landraces and $37 \mathrm{~F}_{1}$ hybrid cultivars released since 1980s, were investigated (Table 1). The landraces were chosen based on the three following criteria: 1) the historical significance to eggplant production in each location of origin; 2) carried traits of particular interests, and 3) being used as important parents in current breeding programs. Seedlings from all accessions were grown in the experimental field of the Institute of Vegetable Crops/Jiangsu Academy of Agricultural Sciences, Nanjing, China. Twenty-two morphological traits were investigated over all 143 accessions following Choudhury's procedure (Choudhury, 1976). Eight groups were clustered based on the matrices of Nei's genetic distance using the PROC CLUSTER/UPGMA in SAS8.0 software (Sneath and Sokal, 1973). Leaf and fruit characteristics of 8 groups are listed in Table 2. 


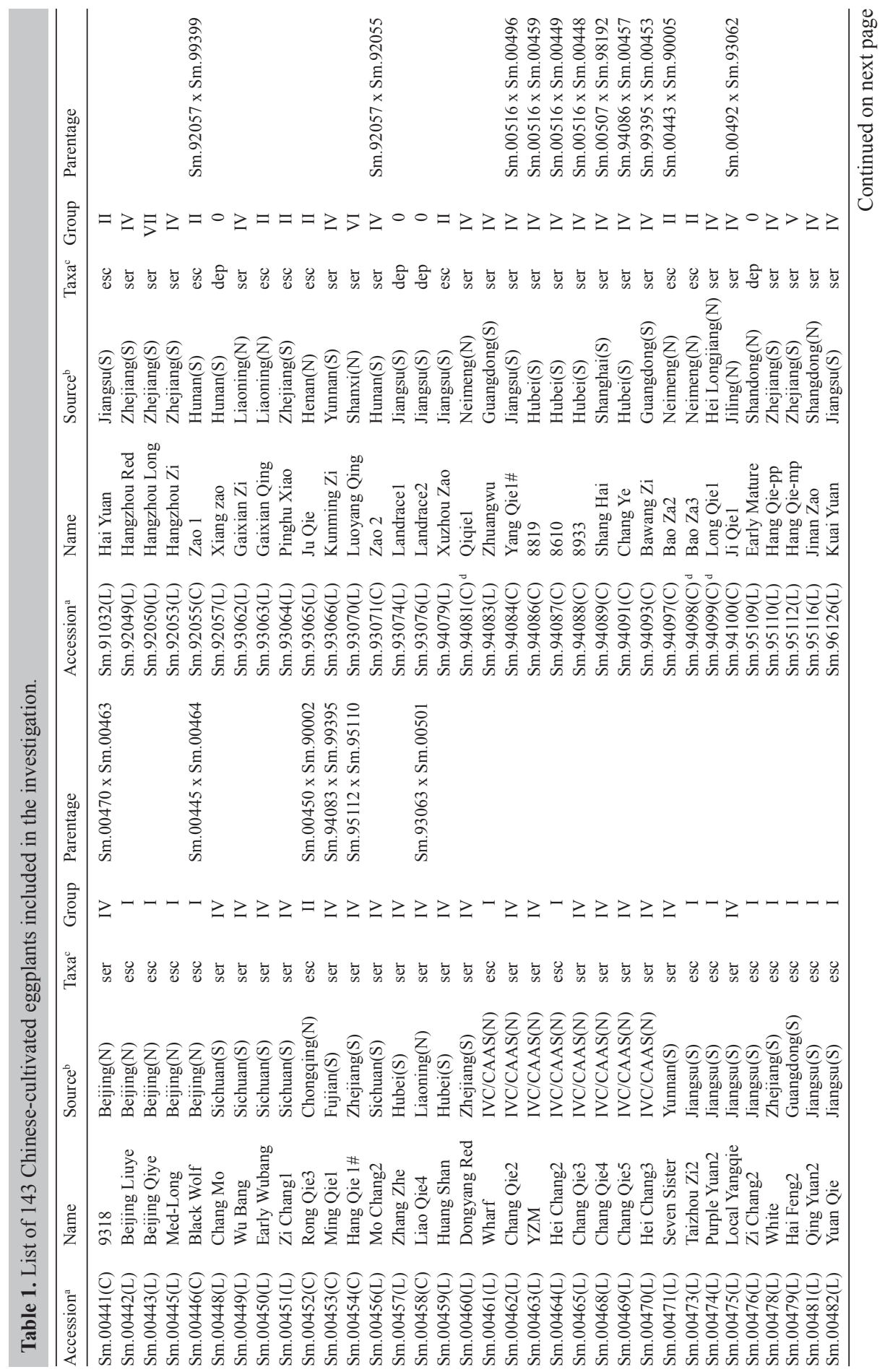


Molecular pedigree diversity in eggplant

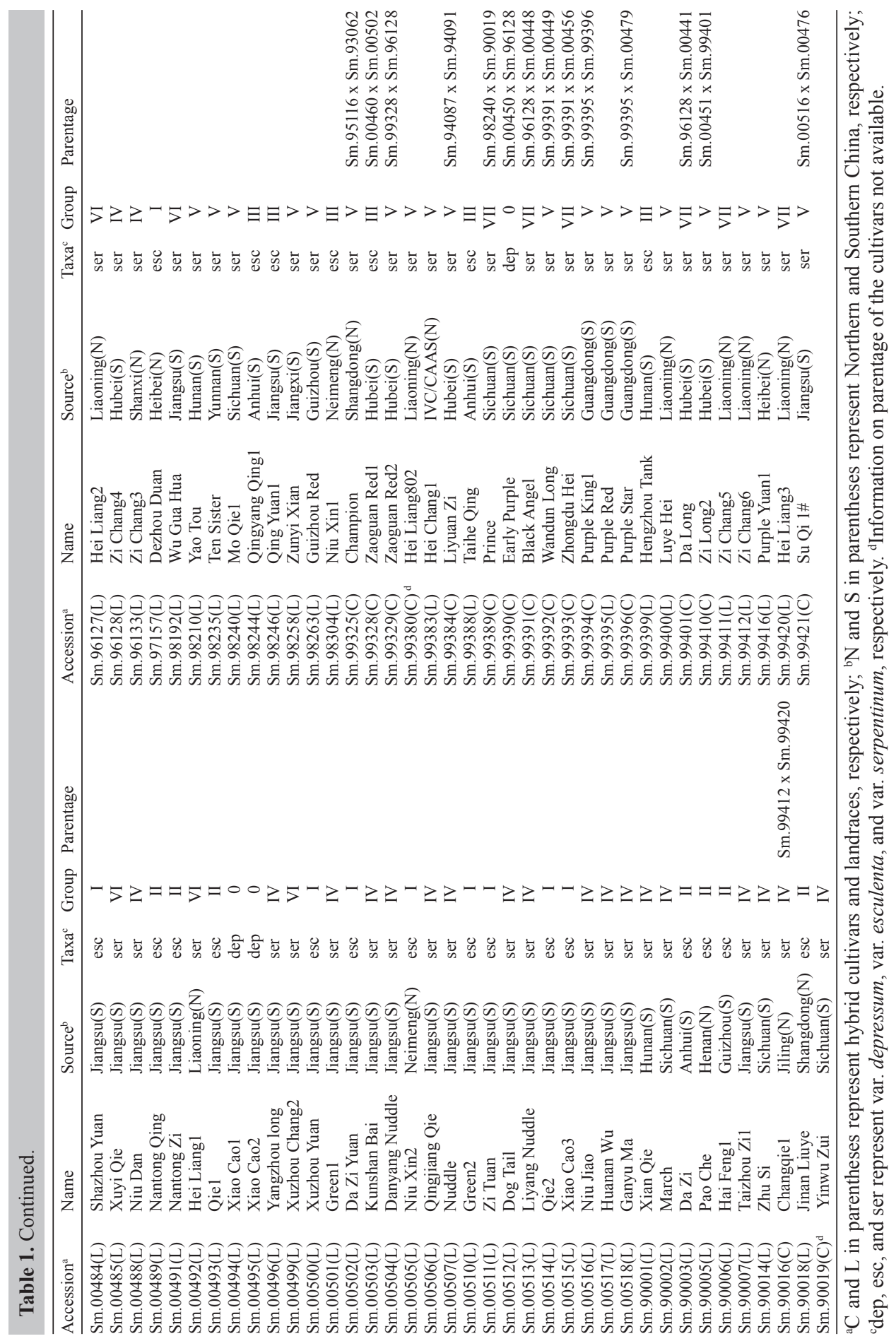


Table 2. Leaf and fruit characteristics of 8 groups as per classification of Yi (2000).

\begin{tabular}{cl}
\hline Groups & Characteristics \\
\hline 0 & No foliage spine, medium to long, bobble to oval, straight fruits \\
I & No foliage spine, medium to long, global to bobble, bend fruits \\
II & No foliage spine, medium to long, global to oval, straight fruits \\
III & Some spiny foliage, medium to long, global to oval, mostly bend fruits \\
IV & No foliage spine, long to very long, bend fruits \\
V & Some spiny foliage, long oval to very long, mostly bend fruits \\
VI & No foliage spine, medium long to long, mostly bend fruits \\
VII & Some spiny foliage, long, straight fruits \\
\hline
\end{tabular}

\section{DNA isolation and RAPD/ISSR protocol}

DNA was extracted from $0.5 \mathrm{~g}$ fresh young leaves following the CTAB procedure (Murray and Thompson, 1980). A total of 280 10-mer RAPD primers and 39 20-mer ISSR primers were randomly chosen and synthesized at Sunshine Co. Ltd., Shanghai, China. Twelve RAPD and five ISSR primers generated the most polymorphic fragments and thus were chosen to amplify across all 143 accessions.

PCR mixtures $(20 \mu \mathrm{L})$ contained the following components/concentrations for RAPDs: 1 U Takara Taq polymerase, 5 pmol primer, $0.225 \mathrm{mM}$ of each dNTP (Takara Co. Ltd.) and 20 ng template genomic DNA and 2.0 $\mu \mathrm{L}$ 10X PCR buffer. Protocol of Zietkiewicz et al. (1994) was adopted for ISSRs. In this case, $20-\mu \mathrm{L}$ aliquots for reaction contained $1 \mathrm{U}$ Taq polymerase, 4 ng gDNA template, 10 pmol primer, $0.225 \mathrm{mM}$ of each dNTP, and $2.0 \mu \mathrm{L} 10 \mathrm{X}$ PCR buffer. DNA amplification was performed in a Programmable Thermal Controller, PTC-100 ${ }^{\mathrm{TM}}$ (MJ Research, Inc.) with a preliminary step of $2 \mathrm{~min}$ at $94^{\circ} \mathrm{C}, 45$ cycles of $30 \mathrm{~s}$ at $94^{\circ} \mathrm{C}, 1 \mathrm{~min} 30$ $\mathrm{s}$ at $40^{\circ} \mathrm{C}$ and $2 \mathrm{~min} 30 \mathrm{~s}$ at $72^{\circ} \mathrm{C}$ and a final step of $7 \mathrm{~min}$ at $72^{\circ} \mathrm{C}$ for RAPD. For ISSRs, an initial step of $5 \mathrm{~min}$ at $94^{\circ} \mathrm{C}$, followed by 40 cycles of $30 \mathrm{~s}$ at $94^{\circ} \mathrm{C}, 45 \mathrm{~s}$ at $52^{\circ} \mathrm{C}$ and $2 \mathrm{~min}$ at $72^{\circ} \mathrm{C}$ and a final 6-min extension at $72^{\circ} \mathrm{C}$.

PCR products were examined by electrophoresis on a $1.5 \%$ agarose gel containing $0.25 \mathrm{ng} / \mathrm{mL}$ ethidium bromide at $4 \mathrm{~V} / \mathrm{cm}$ for $4 \mathrm{~h}$ in TAE buffer. The amplified DNA fragments were observed under the UV Transilluminator (Nippon Gene Co. Ltd., Toyama, Japan).

\section{Data analysis}

RAPD and ISSR fragments were scored for presence (1) or absence (0) of homologous fragments over all accessions. Herein, electropherograms were automatically scored by LabWork $^{\mathrm{TM}}$ Software version 3.02.00 (Gene Co. Ltd.) and validated by two researchers. The condensed distinct fragments were counted. Calculation of COP was based on the assumptions and procedures described by Falconer and Mackay (1996). The proportional contribution of an ancestral germplasm source to a descendent germplasm source is estimated by.

$$
P_{i k}=\frac{\sum_{j=1}^{t} C_{i j} / t}{\sum_{i=1}^{s} \sum_{j=1}^{t} C_{i j} /(s t)},
$$


where $C_{i j}$ is the co-ancestry between the $i^{\text {th }}$ "ancestral" germplasm source and $j^{\text {th }}$ descendent germplasm source belonging to the $k^{\text {th }}$ germplasm group, $i=1,2, \ldots, s ; j=1,2, \ldots, t ; s$ is the number of ancestral germplasm sources, and $t$ is the number of descendent germplasm sources.

Genetic diversity was calculated as: 1) GDI for phenotypic diversity (Shannon, 1948) has recently been frequently applied to analyze molecular data (Xu et al., 1994; Maughan et al., 1996; Paul et al., 1997). This index was defined as $H_{0}=-\Sigma P_{i} L n P_{i}$, where $H_{0}$ is the diversity value of a single band and $P_{\mathrm{i}}$ is the frequency of a RAPD/ISSR band in the entire collection. The average diversity of entire collection was defined as $\mathrm{H}_{\mathrm{a}}=1 / \mathrm{n} \Sigma \mathrm{H}_{0}$, where $n$ is the number of fragments; 2) The average value of all pairwise distances was regarded as a measure of nucleotide polymorphism (nucleotide diversity). Pairwise comparisons between accessions were made using Nei and Li's coefficient (Nei and Li, 1979): $\mathrm{S}_{\mathrm{ij}}=2 \mathrm{~N}_{\mathrm{ij}} /\left(\mathrm{N}_{\mathrm{i}}+\right.$ $\mathrm{N}_{\mathrm{j}}$ ), where $N_{\mathrm{i}}$ represents the number of polymorphic bands of $i^{\text {th }}$ accession, $N_{\mathrm{j}}$ represents the number of polymorphic bands of $j^{\text {th }}$ accession, and $N_{\mathrm{ij}}$ represents the number of shared polymorphic bands between $i^{\text {th }}$ and $j^{\text {th }}$ accessions. In addition, the percentage of polymorphic fragments for each primer was calculated by dividing the number of polymorphic fragments by the total number of fragments generated by that primer in overall accessions.

Canonical discriminant analysis was conducted for distinguishing between groups of eggplant accessions using the PROC DISCRIM in SAS software.

\section{RESULTS}

\section{DNA polymorphism and ISSR/RAPD variations in the Chinese-cultivated eggplant}

Twelve of 280 RAPD primers and 5 of 29 ISSR primers generated polymorphic fragments over 106 landraces and 37 hybrid cultivars, as shown in Table 3. RAPD and ISSR primers yielded 403 and 285 reproducible fragments in landraces and 130 and 87 in hybrid cultivars, respectively. All PCR products ranged from 300 to $3200 \mathrm{bp}$ in size. Of all reproducible fragments, RAPD primers generated 13.9, 14.8 and $14.7 \%$ polymorphic bands in landraces, hybrid cultivars and across all 143 accessions, respectively. Whereas ISSR primers yielded a respective polymorphism of 13.5, 20.7 and 15.3\% in landraces, hybrid cultivars and across all 143 accessions. The number of polymorphic fragments produced by each primer over 106 landraces ranged from 6 by AN11 to 144 by AY13, it was 0 by AN07 to 35 by AY13 over 37 hybrid cultivars. However, AY13 and ISSR-59 generated maximum respective 179 and 95 polymorphic bands overall in 143 accessions. An example of the RAPD and ISSR products over 28 representative accessions is shown in Figure 1. Integrating the polymorphic pattern generated by RAPD and ISSR primers, Jaccard's similarity was calculated in a paired comparison, revealing that the diversity generated by the two types of markers varied from 0.10 to 0.51 .

\section{Subdivision of three morphological varieties included in $S$. melongena $\mathbf{L}$.}

The traditional morphological-based division of $S$. melongena into three varieties, e.g., var. depressum, var. esculenta and var. serpentinum (Choudhury, 1976), was considered as the classification criterion in discriminant analysis (Figure 2). The canonical correlations corresponding to the first two axes were 0.744 and 0.661 , respectively, significantly different 
Table 3. List of polymorphic RAPD and ISSR primers, and their number of fragments generated in landraces and hybrid cultivars.

\begin{tabular}{|c|c|c|c|c|c|}
\hline & \multicolumn{2}{|c|}{ Total fragments } & \multicolumn{2}{|c|}{ Polymorphic fragments } & \multirow[t]{2}{*}{ Total } \\
\hline & Landrace & Hybrid cultivar & Landrace & Hybrid cultivar & \\
\hline \multicolumn{6}{|l|}{ Primer $\left(5^{\prime} \rightarrow 3^{\prime}\right)$} \\
\hline AA07 (CTACGCTCAC) & 283 & 87 & 41 & 18 & 59 \\
\hline AY01 (GTCCACCTCT) & 371 & 103 & 61 & 37 & 98 \\
\hline AY12 (CTGTCGGCGT) & 347 & 113 & 85 & 27 & 112 \\
\hline AY13 (CCGCTCGTAA) & 504 & 175 & 144 & 35 & 179 \\
\hline AC03 (CACTGGCCCA) & 301 & 93 & 23 & 12 & 35 \\
\hline AC04 (ACGGGACCTG) & 603 & 189 & 45 & 21 & 66 \\
\hline AC08 (TTTGGGTGCC) & 599 & 188 & 49 & 23 & 72 \\
\hline AN11 (GTCCATGCAG) & 318 & 90 & 6 & 15 & 21 \\
\hline AN13 (CTTCCAGGAC) & 447 & 167 & 93 & 8 & 101 \\
\hline AN02 (CACCGCAGTT) & 303 & 81 & 21 & 25 & 46 \\
\hline AN07 (TCGCTGCGGA) & 463 & 175 & 77 & 0 & 77 \\
\hline AY07 (GACCGTCTGT) & 297 & 96 & 27 & 9 & 36 \\
\hline RAPD mean & 403 & 130 & 56 & 19 & 75 \\
\hline ISSR-09 ((CTC) 6) & 283 & 93 & 41 & 13 & 54 \\
\hline ISSR-34 ((AG) 8AA) & 295 & 79 & 29 & 26 & 55 \\
\hline ISSR-40 ((ACC) 6) & 415 & 126 & 17 & 14 & 31 \\
\hline ISSR-55 ((TG) 8GG) & 180 & 57 & 36 & 13 & 49 \\
\hline ISSR-59 ((AG) 8GC) & 254 & 80 & 70 & 25 & 95 \\
\hline ISSR mean & 285 & 87 & 39 & 18 & 57 \\
\hline Overall mean & 286 & 87 & 38 & 18 & 56 \\
\hline
\end{tabular}

ISSRs = inter-simple sequence repeats; RAPD = random amplified polymorphic DNA.

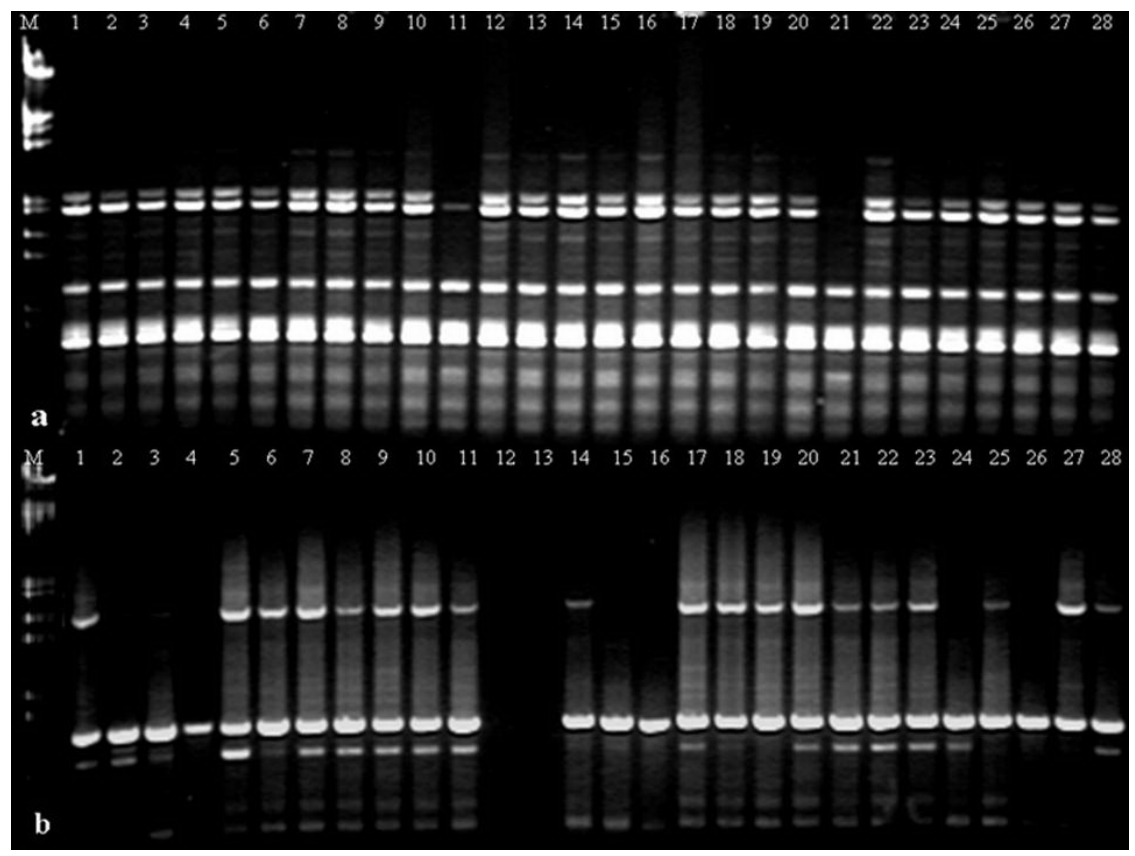

Figure 1. Electrophoresis patterns on 28 representative accessions using a. RAPD-PCR primer AA07 and b. ISSRPCR primer ISSR-09. M is DNA molecular weight marker ( $\lambda \mathrm{DNA} / H i n \mathrm{dIII}+E c o \mathrm{RI})$. Accessions in each lane were arranged from Sm.00442 to Sm.00469. 
from zero $(\mathrm{P}<0.05)$. The separation of three varieties along the first and second canonical variables was mainly attributed to AY13 and AN02, respectively. All 7 accessions in var. depressum demonstrated a considerable divergence from the other two varieties. However, this variety is more close to var. esculenta. There were also considerable overlaps between var. esculenta and var. serpentinum.

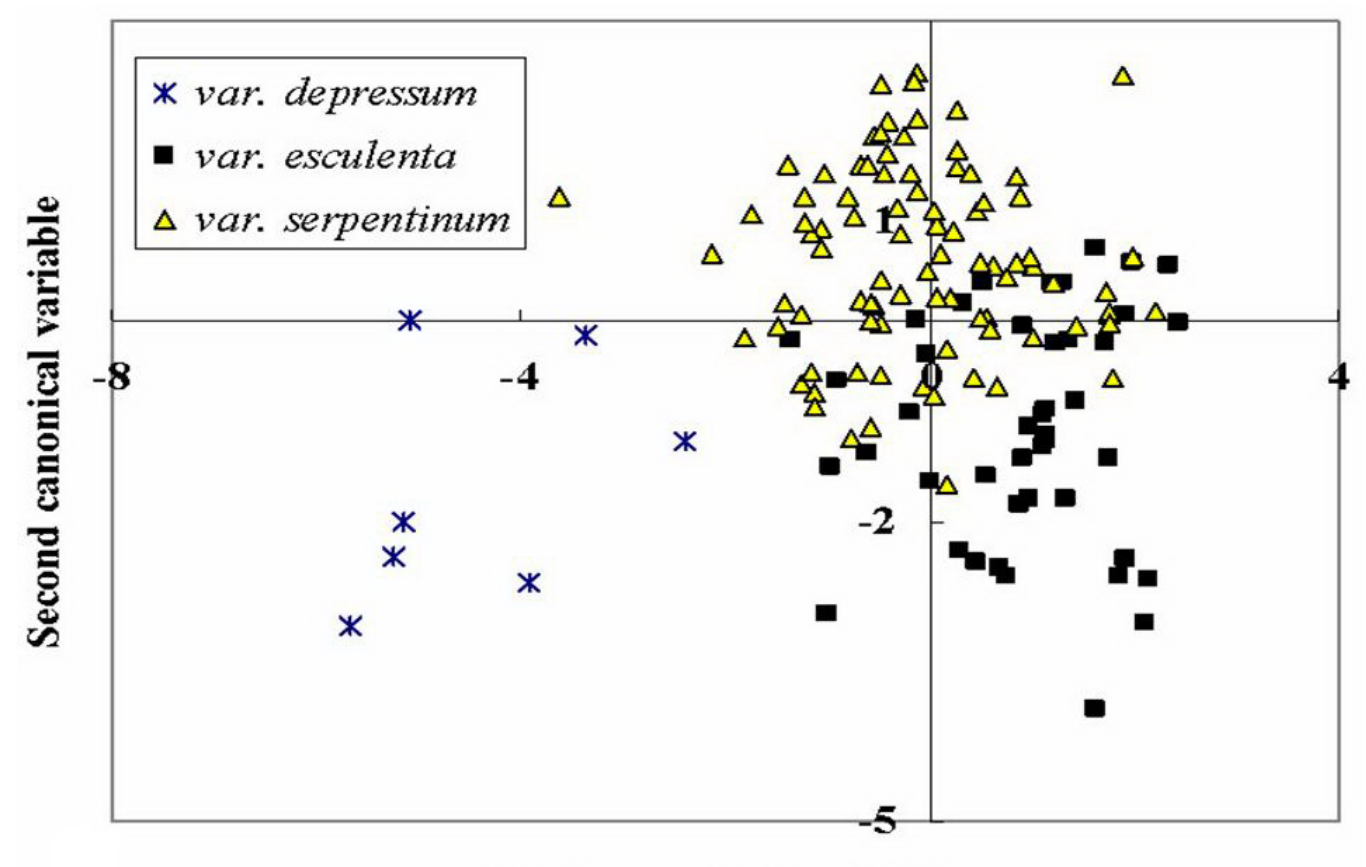

First canonical variable

Figure 2. Canonical discriminant analysis of diversity for molecular data; molecular markers AY13 and AN02 were used as first and second canonical variables, while morphological classification of Solanum melongena into three varieties was set as an initial classification criterion.

A further canonical discriminant analysis was conducted according to previously proposed 8 groups as initial classification criterion (Yi, 2000), as for Figure 3. The canonical correlations $(r)$ of the first and second axes were 0.931 and 0.815 , which significantly deviated from zero at $\mathrm{P}<0.0001$ and $\mathrm{P}<0.0027$, respectively. This explains the respective axis contribution of 49.3 and $15.0 \%$ in the total variation. The original variables that contributed most to the separation along the first and second canonical variables mainly included AN02 and AY13, respectively. All 7 accessions from var. depressum were clustered in "Group 0" and cannot be further subdivided. Whereas, the other two varieties, i.e., var. esculenta and var. serpentinum, were subdivided into 3 and 4 groups, respectively. The three groups in var. esculenta contained 22 (I), 16 (II) and 6 (III) accessions. However, notable overlaps between Groups I and II were observed. In var. serpentinum, Groups IV, V, VI, and VII comprised 59, 20, 6, and 7 accessions, respectively, and all four groups were distinct from each other. 


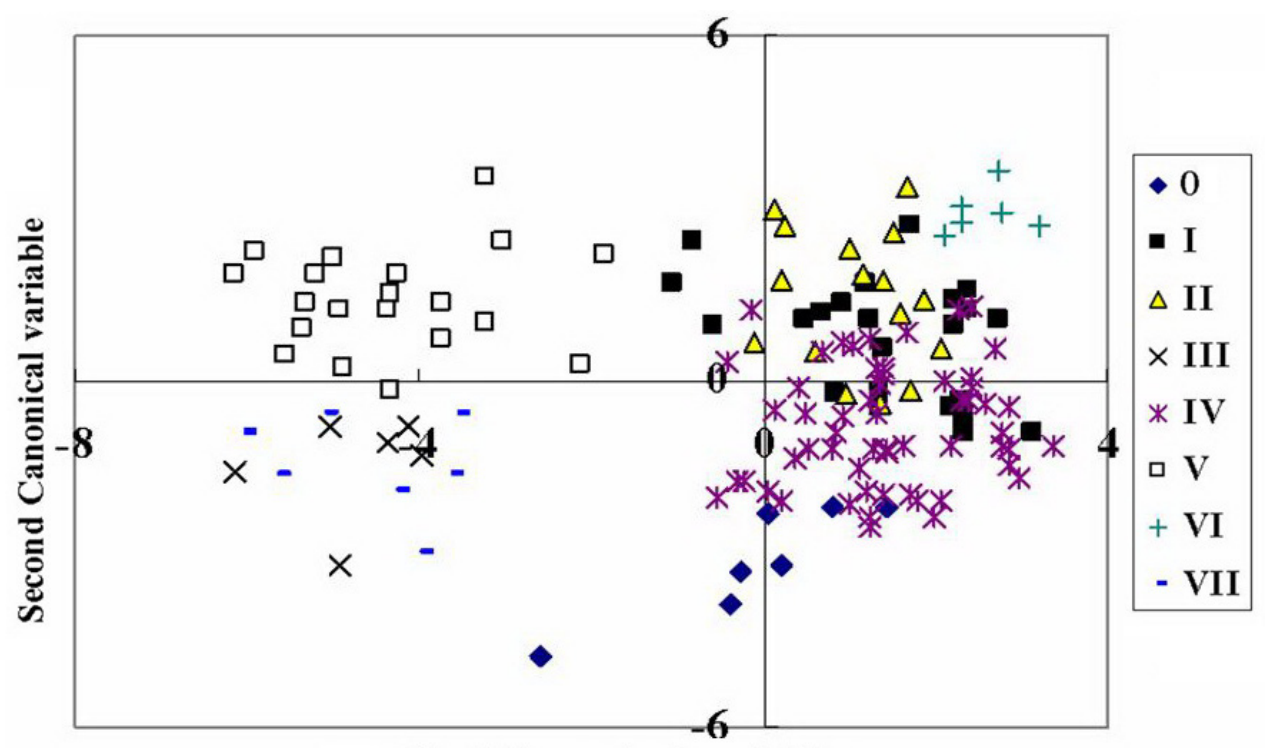

First Canonical variable

Figure 3. Canonical discriminant analysis of diversity over 143 Chinese-cultivated eggplant accessions using ISSR/RAPD marker data with 8 morphological-based groups as an initial classification criterion.

\section{Pedigree diversity pattern in the Chinese-cultivated eggplant collection}

Distinct increments of coefficient of parentage between 1980-1991 and 1992-2003 were observed in the Chinese-cultivated eggplant, as shown in Table 4. In the COP overall, 143 accessions increased from 0.05 to 0.10 in the period from 1980-1991 to 1992-2003. This trend is particularly pronounced in Groups I and V, in which the respective increase in the average COP was observed to be nearly 2- and 3-fold between the two periods. Nevertheless, Groups II, IV and VII displayed only slight changes contemporaneously. It was also observed that northern China-derived accessions demonstrated unchanged COP while southern ones showed a slight increment. Moreover, Table 4 shows twice the increment of proportional contributions (PC), percentage of ancestral accessions, from 3.22 to $6.46 \%$ overall 143 accessions when comparing the aforementioned two periods. Groups I, V and VII showed 2-4-fold increase of PC, contrastingly, Group II showed a drastic decrease and Group IV a slight decrease. The average GDI of landraces was 0.21 , whereas GDI of hybrid cultivars released during 1980-1991 and 1992-2003 was 0.09 and 0.08, respectively. Furthermore, the GDI of hybrid cultivars in var. esculenta decreased $50 \%$ but that of var. serpentinum decreased $33 \%$ when comparing the two periods.

\section{DISCUSSION}

\section{DNA polymorphism and ISSR/RAPD variations in the Chinese-cultivated eggplant}

ISSR primers generated more polymorphic fragments compared to RAPD primers in both landraces and hybrid cultivars, and thus more effective to uncover polymorphism in 
Molecular pedigree diversity in eggplant

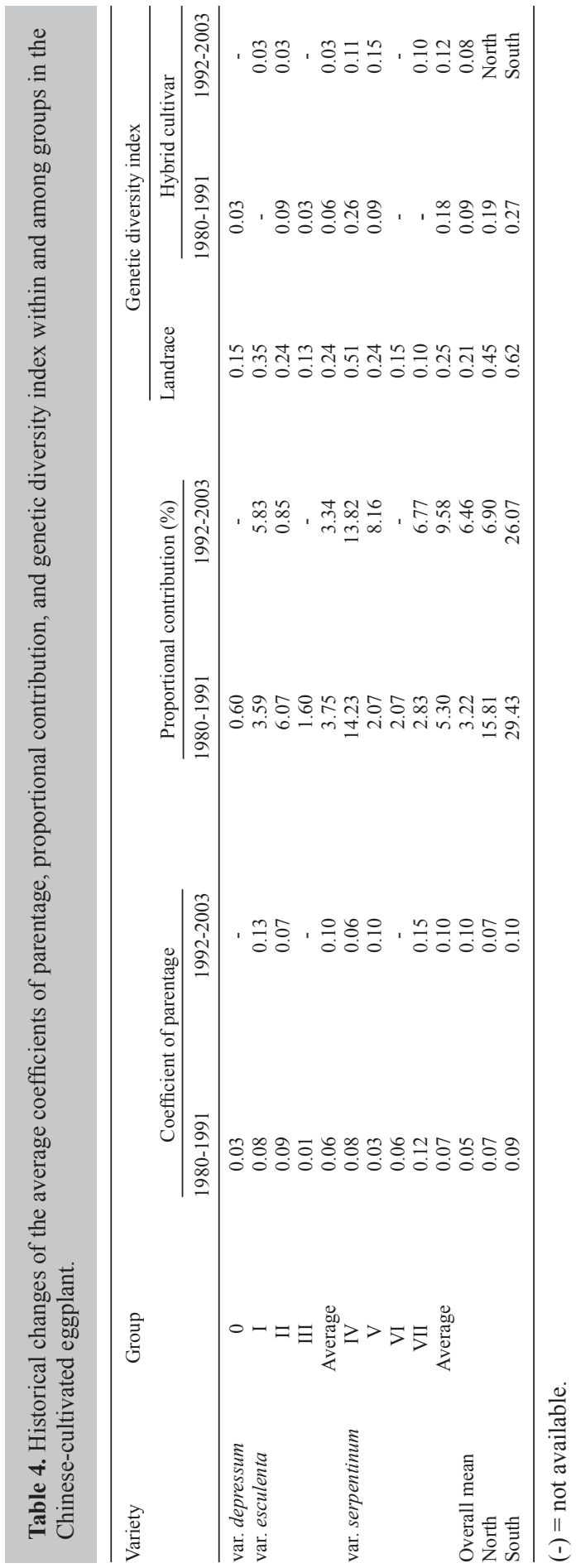


eggplant. This was also observed in several other plant species like wheat (Nagaoka and Ogihara, 1997; Souframanien and Gopalakrishna, 2004) and vigna (Ajibade et al., 2000). Moreover, landraces demonstrated slightly more polymorphisms per primer per accession than hybrid cultivars. This might be explained by the fact that parents of the hybrid cultivars were concentrated in a narrow range of landraces along with the breeding progress. For instance, 12 of 37 hybrid cultivars had both parents derived from Group IV, and 11 hybrid cultivars had one of the parents belonging to Group IV. Intensive crosses within Group IV or related groups in many breeding programs inevitably resulted in a decrement of diversity in released hybrid cultivars. On the contrary, the landraces showed more diversity, which may depend on the fact that the landraces may harbor not only the unique genes in the group it belongs to, but also the allele genes shared with some other groups as well. Our ISSR/RAPD data also indicated that there exists a considerable high genetic homozygosity between var. esculenta and var. serpentinum since overlaps were observed in ISSR/RAPD-based canonical discriminant analysis, and the results agreed with a previous report by Karihaloo et al. (1995). The narrow genetic base was also confirmed by the Jaccard's similarity, which ranged from 0.10 to 0.51 and was lower than barley from 0.16 to 0.65 (Fernández et al., 2002) and maize from 0.31 to 0.64 (Ajmone-Marsan et al., 1998).

\section{Subdivision of three morphological varieties included in S. melongena L.}

Despite the morphological divergence among three varieties of cultivated eggplants (Yi and Yang, 1997; Yi, 2000), current RAPD/ISSR-based canonical discriminant analysis has shown considerable overlaps between var. esculenta and var. serpentinum but completely separated from var. depressum, as in Figure 2, implying a narrow genetic base between the former two varieties but distinct to var. depressum. Nevertheless, some confusion may occur as var. depressum shared a high similarity with var. esculenta at the morphological level but in fact was a distinct variation at the DNA level. The former variety harbors more genetic differences, which are sufficient to separate the two varieties based on patterns of molecular markers; whereas var. esculenta and var. serpentinum demonstrated a more close kinship when compared to var. depressum. In general, our study has provided molecular evidence well in agreement with the previous morphological-based classification of three varieties within cultivated eggplant (Choudhury, 1976). A further subdivision of var. serpentinum into 4 groups was distinctive and a well-matched previous morphologybased grouping (Yi and Yang, 1997; Yi, 2000). However, previous suggested subdivision of 3 groups in var. esculenta can only offer partial support since only Group III was dispersed from Groups I and II, but the latter two groups were overlapped. Furthermore, the separation of Group III apart from Groups I and II was mainly attributed to AN02 marker along the first canonical variable and was not attributable by both ISSR9 and AY13 markers along the second canonical variable; but no markers were able to make a distinctive separation between Groups I and II. The closely related groups implied that alleles might be shared by these groups, and the alleles were distributed evenly among the groups. Nevertheless, the division of Groups IV and VI from Groups $\mathrm{V}$ and VII in var. serpentinum depended on AN02 and AY13 along x- and y-axes, respectively. Notably, no overlaps among the four groups indicated more diversity within var. serpentinum when compared to that of var. esculenta. Molecular data also grouped all accessions in var. depressum into Group 0 . Since this group yielded small and bitter fruit they were therefore seldomly involved in commercial production; as a result the number of accessions in this group is not adequate to reflect the entire diversity of the taxa. However, all of this taxa derived from southern China except 
"Early Mature" (Sm.95109), exhibiting a major pattern of geographic distribution.

\section{Pedigree diversity pattern in the Chinese-cultivated eggplant collection}

There were distinct patterns of diversity among varieties (Table 4). The average COP of elite hybrid cultivars from var. depressum, var. esculenta, and var. serpentinum was as low as $0.03,0.08$, and 0.09 , respectively; implying minor exchanges among gene pools of each variety. This might be due to geographical isolation among the three varieties: most of the hybrid cultivars in var. esculenta were grown in the northern region, whereas var. serpentinum were grown in the southern region, whereas no hybrid cultivar developed from the third variety var. depressum. Moreover, var. serpentinum displayed the highest GDI (0.25) among three varieties, and followed by var. esculenta (0.24) and var. depressum (0.15), implying the diverse genetic base of each variety.

The average COP of accessions derived from northern China was 0.07 in the past 24 years, similar to 0.08 in south of China. The variation of COP between northern and southern China primarily reflects the different frequency of the ancestors in breeding programs in the two regions. The pattern of southern and northern Chinese-cultivated eggplant can be largely explained by the predominant involvement of few landraces with significant regional adaptation and high yield in breeding programs. This is clearly seen in the hybrid cultivar "Su Qi 1\#", a milestone cultivar released in southern China in 1987 with significantly improved yield and agronomic value. "Niu Jiao" (Sm.00516), an elite parent of "Su Qi 1\#", was intensively employed as a co-ancestor in most breeding programs following two decades in southern China, and therefore resulting in a release of a series of hybrid cultivars including "Yang Qie 1\#" (Sm.94084), "8819" (Sm.94086), "8610" (Sm.94087), and "8933" (Sm.94088). Furthermore, "8819" (Sm.94086) and "8610" (Sm.94087) were further used to develop "Chang Ye" (Sm.94091) and "Liyuan Zi" (Sm.99384), respectively. The landrace "Purple Red" (Sm.99395) also played a similar role like "Niu Jiao" (Sm.00516) in southern China. In northern China, the elite landrace "Beijing Qiye" (Sm.00443) has established similar restrictions on the ancestral base before 1990s. More recently the cultivar "Champion" (Sm.99325) was widely used in eggplant production and had a similar impact on diversity patterns. Therefore, we proposed that a new germplasm with desired yield value and diversity from some other gene pools might be introduced to breeding programs both in northern and southern China, offering more opportunity of choice (Polignano et al., 2010).

\section{Impacts of breeding to genetic diversity}

Breeders usually choose elite inbred lines from segregating populations, which is established by crossing two landraces or cultivars with desired and complementary characteristics. However, concentration on few core inbred lines in many breeding programs will result in a decrease of genetic diversity and therefore the released cultivars displaying a high similarity. This is particular evident in breeding programs involving var. esculenta during the past two decades. The increase of average COP between 1980-1991 and 1992-2003 indicated that most of the crosses were concentrated on few parents and manipulated within groups other than among groups (Table 4). Moreover, along with the large scale application of these hybrid cultivars in eggplant production, the genetic diversity of these cultivars decreased 75.0 and $87.5 \%$ during the two periods, respectively. These changes were particularly contributed 
by Group I in this variety. The intensive use of "Beijing Qiye" (Sm.00443) as an ancestor parent and extensive crossing within this group during the past decade caused a $62.4 \%$ increase of PC, and $91.4 \%$ decrease of the genetic diversity during 1992-2003. Since there is a lack of adequate genetic variance, no outstanding hybrid cultivar in this variety has been released until more recently.

The average GDI in var. serpentinum only decreased 28.0 and $52.0 \%$, which is $3-$ and 4-fold higher than that of var. esculenta during the aforementioned two periods, respectively. The major ancestors of cultivars in this variety were concentrated on few landraces; however, more diverse alien cultivars were introduced during 1992 to 2003 and therefore may extend the genetic bases of the eggplant-breeding program. Many of the most successful eggplant hybrid cultivars were derived from crosses between local maternal parent and alien paternal parent. Recent examples after 2003 include "Black Beauty" from USA, "Chanqi Long" from Japan and "Da Dragon" from Korea, three of the most widely used parents in recent breeding.

\section{CONCLUSIONS}

Our ISSR/RAPD data provide molecular evidence supporting previous morphological-based classification of three varieties and subdivision of 8 groups, except Groups I and II in the Chinese-cultivated eggplant. The intensive use of elite parents and extensive crossing within groups have resulted in a decrease of genetic diversity during the past decades. However, the introduction of alien genotypes may extend the genetic bases of eggplant breeding program.

\section{ACKNOWLEDGMENTS}

The authors are grateful to Yuexiang Leng and Fang Xu for their technical assistance. Z. Ali thanks financial assistance from Jiangsu and Punjab sister provinces of China and Pakistan for his visit at Jiangsu Academy of Agricultural Sciences, Nanjing, P.R. China.

\section{REFERENCES}

Ajibade SR, Weeden NF and Chite SM (2000). Inter-simple sequence repeat analysis of genetic relationships in the genus Vigna. Euphytica 111: 47-55.

Ajmone-Marsan P, Castiglioni P, Fusari F, Kuiper M, et al. (1998). Genetic diversity and its relationship to hybrid performance in maize as revealed by RFLP and AFLP markers. Theor. Appl. Genet. 96: 219-227.

Chai XY, Chen SL and Xu W (2010). Using inter-simple sequence repeat markers to analyze the genetic structure of natural Pteroceltis tatarinowii populations and implications for species conservation. Plant Syst. Evol. 285: 65-73.

Choudhury B (1976). Vegetables. 4th Revised edn. National Book Trust, New Delhi.

Cowen RK (1985). Large scale pattern of recruitment by the labrid, Semicossyphus pulcher: causes and implications. $J$. Marine Res. 43: 719-742.

Cox DR and Snell EJ (1989). The Analysis of Binary Data. 2nd edn. Chapman and Hall, London.

Crema S, Cristofolini G, Rossi M and Conte L (2009). High genetic diversity detected in the endemic Primula apennina Widmer (Primulaceae) using ISSR fingerprinting. Plant Syst. Evol. 280: 29-36.

Dreisigacker S, Zhang P, Warburton ML, Van Ginkel M, et al. (2004). SSR and pedigree analyses of genetic diversity among CIMMYT wheat lines targeted to different megaenvironments. Crop Sci. 44: 381-388.

Falconer DS and Mackay TFC (1996). Introduction to Quantitative Genetics. 4th edn. Longman, London.

Fasoula VA and Fasoula DA (2002). Principles underlying genetic improvement for high and stable crop yield potential. Field Crops Res. 75: 191-209. 
Fernández ME, Figueiras AM and Benito C (2002). The use of ISSR and RAPD markers for detecting DNA polymorphism, genotype identification and genetic diversity among barley cultivars with known origin. Theor. Appl. Genet. 104: 845-851.

Han YC, Teng CZ, Wahiti GR, Zhou MQ, et al. (2009). Mating system and genetic diversity in natural populations of Nelumbo nucifera (Nelumbonaceae) detected by ISSR markers. Plant Syst. Evol. 277: 13-20.

Karihaloo JL and Gottlieb LD (1995). Allozyme variation in the eggplant, Solanum melongena L. (Solanaceae). Theor. Appl. Genet. 90: 578-583.

Karihaloo JL, Brauner S and Gottlieb LD (1995). Random amplified polymorphic DNA variation in the eggplant, Solanum melongena L. (Solanaceae). Theor. Appl. Genet. 90: 767-770.

Lester RN and Hasan SMZ (1991). Origin and Domestication of the Brinjal Egg-Plant, Solanum melongena, from $S$. incanum, in Africa and Asia. In: Solanaceae III Taxonomy, Chemistry, Evolution (Hawkes JG, Lester RN, Nees M and Estrada N, eds.). Royal Botanic Gardens, Kew, 369-387.

Lu X, Liu L, Gong Y, Zhao L, et al (2009). Cultivar identification and genetic diversity analysis of broccoli and its related species with RAPD and ISSR markers. Sci. Hort. 122: 648 -645.

Mace ES, Lester RN and Gebhardt CG (1999). AFLP analysis of genetic relationships among the cultivated eggplant, Solanum melongena L., and wild relatives (Solanaceae). Theor. Appl. Genet. 99: 626-633.

Martin FW and Rhodes AM (1979). Subspecific grouping of eggplant hybrid cultivars. Euphytica 28: 367-383.

Maughan PJ, Saghai Maroof MA, Buss GR and Huestis GM (1996). Amplified fragment length polymorphism (AFLP) in soybean: species diversity, inheritance, and near-isogenic line analysis. Theor. Appl. Genet. 93: 392-401.

Murray MG and Thompson WF (1980). Rapid isolation of high molecular weight plant DNA. Nucleic Acids Res. 8: 43214325.

Nagaoka T and Ogihara Y (1997). Applicability of inter-simple sequence repeat polymorphisms in wheat for use as DNA markers in comparison to RFLP and RAPD markers. Theor. Appl. Genet. 94: 597-602.

Nei M and Li WH (1979). Mathematical model for studying genetic variation in terms of restriction endonucleases. Proc. Natl. Acad. Sci. U. S. A. 76: 5269-5273.

Paul S, Wachira FN, Powell W and Waugh R (1997). Diversity and genetic differentiation among populations of Indian and Kenyan tea (Camellia sinensis (L) O. Kuntze) revealed by AFLP markers. Theor. Appl. Genet. 94: 255-263.

Polignano G, Uggenti P, Bisignano V and Gatta CD (2010). Genetic divergence analysis in eggplant (Solanum melongena L.) and allied species. Genet. Resour. Crop Evol. 57: 171-181.

Sakata Y and Lester RN (1997). Chloroplast DNA diversity in brinjal eggplant (Solanum melongena L.) and related species. Euphytica 97: 295-301.

Shannon CE (1948). A mathematical theory of communication. Bell Syst. Tech. J. 379-423, 623-659.

Sneath PHA and Sokal RR (1973). Numerical Taxonomy: The Principles and Practice of Numerical Classification. Freeman, San Francisco.

Sneller CH (1994). Pedigree analysis of elite soybean lines. Crop Sci. 34: 1515-1522.

Souframanien J and Gopalakrishna T (2004). A comparative analysis of genetic diversity in blackgram genotypes using RAPD and ISSR markers. Theor. Appl. Genet. 109: 1687-1693.

Xu WW, Sleper DA and Krause GF (1994). Genetic diversity of tall fescue germplasm based on RFLPs. Crop Sci. 34: 246-252.

Yi JX (2000). A numerical classification on eggplant germplasm. Acta Hort. Sinica 27: 345-350.

Yi JX and Yang QY (1997). A study on genetic distance and cluster analysis of quantitative characters among parents in eggplant. Jiangsu J. Agri. Sci. 13: 40-43.

Zhang LJ and Dai SL (2009). Genetic variation within and among populations of Orychophragmus violaceus (Cruciferae) in China as detected by ISSR analysis. Genet. Res. Crop Evol. 57: 55-64.

Zhou X, Carter TE, Cui Z, Miyazaki J, et al. (2000). Genetic base of Japanese soybean hybrid cultivars released during 1950 to 1988. Crop Sci. 40: 1794-1802.

Zietkiewicz E, Rafalski A and Labuda D (1994). Genome fingerprinting by simple sequence repeat (SSR)-anchored polymerase chain reaction amplification. Genomics 20: 176-183.3. 